\title{
SEMIUNIFORM SPACES AND TOPOLOGICAL HOMEOMORPHISM GROUPS
}

\author{
R. V. FULLER
}

\begin{abstract}
A well-known sufficient condition that a group of homeomorphisms, $H$, from a topological space $X$ onto itself be a topological group relative to the topology of pointwise convergence is that $X$ be uniformizable and $H$ be equicontinuous.

In this paper we prove an analogous condition in which the space $X$ is assumed to be only regular instead of completely regular (uniformizable). This is accomplished by means of the concepts of semiuniformity and semiequicontinuity introduced here.
\end{abstract}

1. Introduction. Let $H$ be a topological space and $H$ a group of homeomorphisms of $X$ onto $X$. It is of interest to know when $H$ with the point-open topology (i.e., topology of point-wise convergence) is a topological group. A well-known sufficient condition is that $X$ be a uniform space and $H$ be equicontinuous. (See Bourbaki $[1$, p. 305, Corollary].)

In this paper we offer an analogous condition (Theorem 6) which requires a less restricted setting than a uniform space (i.e., completely regular space), namely, a regular space. To accomplish our purpose, in §2, we introduce and relate to more standard notions, the notions of semiuniform space and semiequicontinuity which may be of interest in other applications. In $\$ 3$ we prove the theorem under discussion and end with an example showing semiequicontinuity cannot be replaced by even continuity in the present application.

The reader is referred to Kelley [4] for definitions not given here. If $F$ is a collection of functions from $X$ to $Y,(p, V)$ where $p$ is in $X$ and $V$ a subset of $Y$ denotes all functions in $F$ which take on a value in $V$ at $p$.

\section{Semiuniformities and semiequicontinuity.}

Definition 1. Let $Y$ be a topological space. A collection $U^{*}$ of two-element open covers of $Y$ is said to be a semiuniformity for $Y$ if for each point $q$ in $Y$ and each neighborhood $V$ of $q$ there is a $\left\{V_{1}, V_{2}\right\}$ in $V^{*}$ such that $q \in V_{1} \subset V$ and $X-V_{2}$ is a neighborhood of $q$.

The pair $\left(Y, V^{*}\right)$ where $Y$ is a topological space and $V^{*}$ is a semiuniformity for $Y$ will be called a semiuniform space.

REMARK. One may easily show that a topological space has a semiuniformity if and only if it is regular.

Received by the editors February 16, 1970.

AMS 1969 subject classifications. Primary 5401; Secondary 5428.

Key words and phrases. Semiuniformity, semiequicontinuity, uniform semiuniformity, semitopological group. 
THEOREM 1. Let $(Y, B)$ be a uniform space. Then the collection of all two-element uniform open covers of $Y$ is a semiuniformity for $Y$.

Proof. Let $q$ be in $Y$ and $V$ be a neighborhood of $q$. Then there is an open $B_{1}$ in $B$ such that $B_{1}(q) \subset V$. There is an open, symmetric $B_{2}$ in $B$ such that $B_{2} \circ B_{2} \circ B_{2} \subset B_{1}$. It may be seen that $\left\{B_{1}(q), \cup\left\{B_{2}(y): y \notin B_{2} \circ B_{2}(q)\right\}\right\}$ is a uniform open cover which satisfies Definition 1 relative to $V$ and $q$.

DEFINITION 2. The semiuniformity of the preceding theorem will be called the uniform semiuniformity.

Definition 3. Let $F$ be a family of functions from a topological space $X$ to semiuniform space $\left(Y, V^{*}\right) . F$ is semiequicontinuous if for each $v$ in $v^{*}$ there is an open covering $\mathcal{u}$ of $X$ such that $u$ refines $f^{-1}(\mathcal{V})$ for each $f$ in $F$.

REMARK. It is an easy consequence of the definitions that if $F$ is an equicontinuous family of functions from a topological space $X$ to a uniform space $Y$ then $F$ is semiequicontinuous relative to the uniform semiuniformity.

THEOREM 2. If $F$ is a family of functions from a topological space $X$ to a Hausdorff semiuniform space $\left(Y, V^{*}\right)$ which is compact relative to a jointly continuous topology then $F$ is semiequicontinuous.

Proof. Since the point-open topology is Hausdorff and smaller than the given topology, it must coincide with the given topology.

Let $\left\{V_{1}, V_{2}\right\}$ be in $v^{*}, p$ in $X$, and $q$ in $Y$. Then $q$ is in $V_{i}(i=1$ or 2$)$ and so there is a closed neighborhood of $q, V_{i q}$, which is a subset of $V_{i}$. Letting $K_{i p q}$ denote $\left(p, V_{i q}\right), K_{i p q}$ is a subset of $\left(p, V_{i}\right)$ and a closed hence a compact subset of $F$.

Let $P: F \times X \rightarrow Y$ be defined by $P(f, x)=f(x)$. Then by the Wallace Theorem (see Kelley [4, p. 142]) and joint continuity there is an open neighborhood $W_{i p q}$ of $K_{i p q}$ and an open neighborhood $U_{i p q}$ of $p$ such that $P\left(W_{i p q} \times U_{i p q}\right) \subset V_{i}$ since $V_{i}$ is a neighborhood of $P\left(K_{i p q} \times\{p\}\right)$.

Now $W_{p}=\left\{W_{i p q}: q \in Y, i=1,2\right\}$ is an open cover of $F$ for each $p$ in $X$. Hence $\mathscr{W}_{p}$ contains a finite subcover $W_{p}^{\prime}$. Let $\mathcal{u}_{p}$ $=\left\{U_{i p q}: W_{i p q} \in W_{p}^{\prime}\right\}$. Then $\mathcal{u}=\left\{\cap \mathfrak{u}_{p}: p \in X\right\}$ is an open cover of $X$.

Let $f$ be in $F$ and $\cap u_{p}$ in $u$. Then $f$ is in some $W_{i p q}$ in $W_{p}^{\prime}$. Thus $f\left(U_{i p q}\right) \subset V_{i}$ so $f\left(\cap u_{p}\right) \subset V_{i}$.

Therefore $U$ refines $f^{-1}\left(\left\{V_{1}, V_{2}\right\}\right)$ for each $f$ in $F$.

THEOREM 3. If $F$ is a semiequicontinuous family of functions from a topological space $X$ to a semiuniform space $\left(Y, V^{*}\right)$ then $F$ is evenly continuous. 
Proof. Let $p$ be in $X, q$ in $Y$, and $V$ be an open neighborhood of $q$. Let $\left\{V_{1}, V_{2}\right\}$ be a member of $v^{*}$ such that $q \in V_{1} \subset V$ and $X-V_{2}$ is a neighborhood of $q$.

Since $F$ is semiequicontinuous there is an open cover $u$ of $X$ which refines $\left\{f^{-1}\left(V_{1}\right), f^{-1}\left(V_{2}\right)\right\}$ for all $f$ in $F$. Let $U$ be a member of $u$ which contains $p$. If $f(p)$ is in $Y-V_{2}$ then $U \llbracket f^{-1}\left(V_{2}\right)$ so $U \subset f^{-1}\left(V_{1}\right)$, which is to say, $f(U) \subset V_{1} \subset V$. Therefore $F$ is evenly continuous.

REMARK. With Theorems 2 and 3 one could evidently prove an Ascoli Theorem similar to Kelley's [4, p. 236]. See Kaul [3] for other similar results.

\section{Topological homeomorphism groups.}

THEOREM 4. Let $F$ be a family of one-to-one functions of a semiuniform space $\left(Y, V^{*}\right)$ onto itself. If $F^{-1}$ is semiequicontinuous then the mapping $Q: F \rightarrow F^{-1}$ defined by $Q(f)=f^{-1}$ is continuous relative to the point-open topologies on $F$ and $F^{-1}$.

Proof. Let $(p, V)$ where $V$ is open be a neighborhood of $g^{-1}$ in $F^{-1}$. There is a member $\left\{V_{1}, V_{2}\right\}$ of $v^{*}$ such that $g^{-1} p \in V_{1} \subset V$ and $X-V_{2}$ is a neighborhood of $g^{-1} p$.

Since $F^{-1}$ is semiequicontinuous there is an open cover $\mathcal{U}$ of $X$ such that $\mathcal{U}$ refines $\left\{f\left(V_{1}\right), f\left(V_{2}\right)\right\}$ for all $f$ in $F$. Let $U$ be a member of $\mathcal{u}$ which contains $p$.

Consider the neighborhood of $g,\left(g^{-1} p, U\right)$. If $f$ is in $\left(g^{-1} p, U\right)$ then $f\left(g^{-1} p\right)$ is in $U$ and since $f\left(g^{-1} p\right)$ is not in $f\left(V_{2}\right), U \nsubseteq f\left(V_{2}\right)$. Hence $U \subset f\left(V_{1}\right)$ so $f^{-1}(U) \subset V_{1} \subset V$. Thus

$$
Q\left(g^{-1} p, U\right)=\left\{f^{-1}: f\left(g^{-1} p\right) \in U\right\} \subset\left\{f^{-1}: f^{-1}(U) \subset V\right\} \subset(p, V) .
$$

Therefore $Q$ is continuous.

THEOREM 5. Let $F$ be a semigroup (under composition) of continuous functions from a topological space $X$ into itself. If the point-open lopology on $F$ is jointly continuous then composition is continuous relative to the point-open topology.

Proof. Let $f_{a}$ and $g_{a}$ be nets converging point-wise to $f$ and $g$ respectively. By joint continuity, for any $x$ in $X f_{a}\left(g_{a}(x)\right)$ converges to $f(g(x))$, i.e., $f_{a} \circ g_{a}$ converge point-wise to $f \circ g$. Therefore composition is continuous.

THEOREM 6. If $H$ is a semiequicontinuous group of homeomorphisms (or left) automorphisms is semiequicontinuous relative to a semiuniformity for $G$ then $G$ is a topological group. 
Proof. Since by Theorem $3, H$ is evenly continuous and it is well known that in this case the point-open topology is jointly continuous (see Kelley [4, p. 235, Theorem 19]), it follows from Theorem 5 that composition is continuous. By Theorem 4 , inversion is continuous, so $H$ is a topological group.

Definition 3. Let $G$ be a group and $\mathscr{L}$ a topology on $G$. Then $(G, \mathcal{L})$ is a semitopological group if for each $g$ in $G$ the functions of $G$ to $G$ defined by $x \rightarrow x g$ and $x \rightarrow g x$ (i.e., the right and left automorphisms) are continuous. (See Husain [2, p. 27, Definition 1].)

THEOREM 7. If $G$ is a semitopological group whose group of right (or left) automorphisms is semiequicontinuous relative to a semiuniformity for $G$ then $G$ is a topological group.

Proof. $G$ is isomorphically homeomorphic to the group of right automorphisms with the point-open topology (see Husain [2, p. 32, Theorem 4]). Therefore $G$ is a topological group by Theorem 6 .

ExAmple. Let $R$ be the group of reals under addition with the topology whose base is all intervals of the form $[a, b)$. Then addition is continuous so $R$ is in particular a semitopological group. However inversion (taking negatives) is not continuous so although the topology is regular and has semiuniformities, the right automorphisms can be semiequicontinuous with respect to none by Theorem 7 (this can be seen directly without much difficulty).

The right automorphisms are evenly continuous. Let $[q, q+e)$ be a neighborhood of $q$ and $p$ be in $R$. Then one can show that if $r_{a}(p)$ $=p+a$ is in $[q, q+(e / 2))$ then $r_{a}[p, p+(e / 2)) \subset[q, q+e)$.

We conclude that we cannot replace semiequicontinuity by even continuity in Theorems 6 or 7 .

\section{REFERENCES}

1. Nicolas Bourbaki, Elements of mathematics. General topology. Part 2, Hermann, Paris and Addison-Wesley, Reading, Mass., 1966. MR 34 \#5044b.

2. Taqdir Husain, Introduction to topological groups, Saunders, Philadelphia, $\mathrm{Pa}$., 1966. MR 34 \#278.

3. S. K. Kaul, Compact subsets in function spaces, Canad. Math. Bull. 12 (1969), 461-466.

4. John L. Kelley, General topology, Van Nostrand, Princeton, N. J., 1955. MR $16,1136$.

University of South Carolina, Columbia, South Carolina 29208 